\title{
The effect of low-frequency repetitive transcranial magnetic stimulation (rTMS) on the treatment of aphasia caused by cerebrovascular accident (CVA)
}

\author{
Manouchehr Ilkhani ${ }^{1}$, Hassan Shojaie Baghini ${ }^{2}$, Gohar Kiamarzi ${ }^{3}$, Alipasha Meysamie ${ }^{4}$, Parvin Ebrahimi ${ }^{5 *}$
}

Received: 29 Sep 2016

Published: 27 Dec 2017

\begin{abstract}
Background: Aphasia is a common outcome of Cerebrovascular Accident (CVA) in which clinical interventions have limited effectiveness. Some evidence suggests that noninvasive stimulation of the brain can have beneficial effects in the treatment of CVA induced aphasia. In patients with motor aphasia, repetitive Transcranial Magnetic Stimulation (rTMS) is used to facilitate long-term improvement in speech ability. Since identifying effective methods for treating CVA induced aphasia can be very important in subsequent decision-making and treatment interventions, the objective of this study was to evaluate the effect of low-frequency TMS in Broca's area in the right hemisphere on the treatment of CVA induced motor aphasia.

Methods: This clinical trial enrolled 24 patients with a clinical diagnosis of motor aphasia caused by CVA using convenient sampling. In this study, the effect of stimulation of Broca's area in the right hemisphere was examined by low-frequency rTMS (one Hz) on aphasia caused by CVA. To conduct verbal fluency test in patients, their correct responses to the selected images before and after rTMS during a certain time were recorded and compared by non-parametric Wilcoxon test using SPSS16 and the significance level was considered $<0.05$. Registration ID of this research in IRCT is IRCT2014052417814N1.

Results: The study findings suggested a significant difference between Wilcoxon test results of patients before and after rTMS $(\mathrm{z}=-4.401)$, and it was found that using low-frequency rTMS in the right hemisphere was effective on improving dysarthria in the study population with 95 percent confidence interval $(\mathrm{p}<0.001)$.

Conclusion: According to the findings, low-frequency rTMS has the potential to be considered as a treatment for patients with nonfluent aphasia caused by CVA.
\end{abstract}

Keywords: Repetitive Transcranial Magnetic Stimulation (rTMS), Aphasia, Cerebrovascular Accident (CVA)

Copyright $\subseteq$ Iran University of Medical Sciences

Cite this article as: Ilkhani M, Shojaie Baghini H, Kiamarzi G, Meysamie A, Ebrahimi P. The effect of low-frequency repetitive transcranial magnetic stimulation (rTMS) on the treatment of aphasia caused by cerebrovascular accident (CVA). Med J Islam Repub Iran. 2017 (27 Sep);31:137. https://doi.org/10.14196/mjiri.31.137

\section{Introduction}

Aphasia and cognitive impairment are subsequent outcomes of Cerebrovascular Accident (CVA) (1). Each year, almost 80,000 people who survive after CVA develop aphasia; currently more than one million Americans are living with CVA induced aphasia (2). Aphasia occurs in 21-38 percent of acute CVA and shows more deaths, mor-

\section{Corresponding author: Dr Parvin Ebrahimi, parvin_eb@yahoo.com}

1. Loghman Hakim Hospital, Shahid Beheshti University of Medical Sciences, Tehran, Iran.

2. School of Medicine, Iran University of Medical Sciences, Tehran, Iran.

3. Dr. Ilkhani Electroneurodiagnostic Center, Tehran, Iran.

4. Community and Preventive Medicine Department, Medical Faculty, Tehran University of Medical Sciences, Tehran, Iran.

5. Health Management and Economics Research Center, Iran University of Medical Sciences, Tehran, Iran, \& Department of Health Services Management, School of Health Management and Information Sciences, Iran University of Medical Sciences, Tehran, Iran. bidity and the use of health care resources (3). The findings of a study implemented in Iran showed that 22.7 percent of the sample inpatients after CVA suffered from aphasia (4).

Aphasia can be caused by CVA and in particular through damage to a complex network of cortical and sub-

$\uparrow$ What is "already known" in this topic:

According to some studies, the effectiveness of low-frequency rTMS on the treatment of stroke-induced aphasia have been confirmed, while it is suggested that further studies are necessary to prove this effect. Also, few studies have not come to this result that rTMS is effective in the treatment of the aphasia.

\section{$\rightarrow$ What this article adds:}

Considering the conditions of health status and healthcare system of Iran, choosing a proper therapeutic approach and evaulation of the effectiveness of low-frequency rTMS on the treatment of post-stroke aphasia is important. 
cortical structures perfused by middle cerebral artery (MCA) in the left hemisphere $(5,6)$. Most patients who develop aphasia in acute stroke show a degree of spontaneous recovery, especially during the first 2-3 months after the attack. However, after the acute stage of stroke, a degree of chronic verbal deficit remains in most aphasia patients (5).

Sustainable aphasia is a common and often devastating outcome of dominant cerebral hemisphere stroke in which the effectiveness of clinical interventions is limited. Recent evidence suggests that electric and magnetic stimulation of human motor cortex from skull may improve different mental abilities after CVA. Repetitive Transcranial Magnetic Stimulation (rTMS) technique has shown promising results in motor function (7).

RTMS is a noninvasive and safe method used for inducing or enhancing nerve regeneration in brain: a limited but growing set of evidence suggests that noninvasive stimulation of the brain can have beneficial effects in the treatment of aphasia caused by a stroke (5).

Regarding the use of repetitive TMS in the treatment of aphasia, previous studies by Naeser, et al. (8-9) and Hamilton, et al. $(5,7)$ and others have mainly been based on stimulation of the right inferior frontal cortex in the inferior frontal gyrus (IFG) with low frequency (1 Hz per second), and have reported favorable results in speech improvement.

In a small group of patients with motor aphasia, improvement occurred in motor-language function 10 days after using low-frequency rTMS (1 Hz, inhibitor). So far, speech improvement has been reported mainly for picture naming in case studies after months following stimulation in small samples of patients (for example, 4 patients) (10).

Although it is thought that speech improvement is less likely following a year after stroke, studies show that when an intervention is implemented for patients with chronic aphasia, improvement is possible even for such patients $(11,12)$. These patients require additional reconstructive treatments that will enable them to return to the society as active members. According to some studies, rTMS induced changes in neuronal networks can be continued with improved language functions more than the actual period of stimulation. However, the safety and effectiveness of stimulated rTMS used directly on the areas of stroke and adjacent areas is unclear. In addition, the initial reports of possible complications associated with rTMS led to the development of safety rules on the intensity and frequency of stimulation, duration, the number of trains and the interval between them (11). On the other hand, some previous studies have found inconsistencies about the effect of rTMS on stroke-induced aphasia (13).

According to the literature, most of the studies about the effects of rTMS on the treatment of aphasia caused by Cerebrovascular Accident (CVA) were implemented in developed countries and further studies were suggested to more likely confirm the effectiveness of low-frequency rTMS on the treatment of stroke-induced aphasia $(14,15)$. Besides, no clinical trials in this field have been yet conducted in Iran, considering the conditions of health status and healthcare system of this developing country. Also, aphasia needs to be addressed due to its prevalence and importance, and since identifying effective methods on the treatment of stroke-related aphasia can have an important role in future decision making and treatment interventions, this study aimed to investigate the effect of low-frequency repetitive Transcranial Magnetic Stimulation (rTMS) in Broca's area in the right hemisphere on the treatment of aphasia due to CVA.

\section{Methods}

This clinical trial enrolled patients with a clinical diagnosis of aphasia caused by Cerebrovascular Accident (CVA) who attended Loghman Hakim Hospital and a specialty clinic located in Tehran, Iran in 2014. Inclusion and exclusion criteria of the study were as follows:

1) Absolute right handedness

2) At least a year past the diagnosis of CVA (stroke)

3) Patients with Broca's motor aphasia

4) Persian speaking and at least primary education

Based on past experiences and estimates, a few patients with this disease were available. A total of 24 patients included in the study. A non-probable, convenient sampling was used to select qualified patients. They signed the consent form after the approval of Ethics Committee. Besides, patients' privacy and confidentiality of the information was observed in this study with regard to the Helsinki Declaration of 1968.

In order to ensure the effectiveness of the device in the present study, all patients were examined one year after the stroke and passing the progressive recovery time of the patients without any intervention until one year.

The study group included 24 patients received lowfrequency stimulation $(1 \mathrm{~Hz})$ for ten minutes in Broca's area in the right hemisphere $(\mathrm{RH})$ for ten rTMS sessions. Although the speech center is located in the left hemisphere of right-handed persons, we stimulated the right side of their brain. The reason is that stimulation of the right hemisphere with the frequency of $1 \mathrm{~Hz}$ per second has inhibitory effects. Moreover, the recessive hemisphere (right side) has its own inhibition on the damaged speech center of the left side. Therefore, with this inhibitory effect of the recessive hemisphere, the dominant side will be released. It should be noted that physical examination was performed by neurophysiologists.

Verbal test was performed using still images of everyday objects that were not influenced by cultural, ethnic, and educational differences with no synonyms. Ten seconds was considered for each image. Persian version of Wechsler test was also used to assess vocabulary comprehension. In this test, the number of mentioned objects out of the total number (the percentage of correct answers in the determined time) was recorded and compared by a neurophysiologist before and after treatment protocol, and the number of correct answers was calculated.

To do a blind study, the examiner was not aware of whether the patients received rTMS or not. The data were collected in three separate forms, including a checklist for rTMS sessions and two checklists for verbal tests before and after treatment with rTMS. 
As the sham device as a standard placebo for TMS was not available in Iran, a control group could not be enrolled in this study. It is considered as a limitation of this study. Therefore, for each patient the results of rTMS before and after the treatment were compared to evaulate the effect of the intervention.

There was no missing data in this study and none of the patients were excluded. Descriptive statistics (including frequency, percentage, mean and standard deviation) were used to describe the results. Non-parametric Wilcoxon test was used to compare patients' response before and after stimulation with rTMS. The statistical significance level was considered 0.05 . Statistical tests were performed using SPSS version 16.

\section{Results}

According to data collected, 70.8 percent of patients $(n=17$ out of 24$)$ were men and the rest $(29.2 \%)$ were women. The majority of participants $(62.5 \%)$ were $50-69$ years old and the mean age of participants was 63.7 $( \pm 17.5)$ years old. Half of the participants had right hemiplegia.

Most of the participants $(\mathrm{n}=15)$ had under diploma degree, $16.7 \%$ of them had a diploma, and the remained had master's degree (Table 1).

According to the findings, from 10 images shown to 24 patients before and after treatment protocol using rTMS, $20 \%$ of patients mentioned the name of images correctly before treatment, while $40.8 \%$ of patients did so after treatment (Diagram 1).

Due to the lack of normal distribution of patients' correct responses before and after treatment with rTMS, nonparametric tests is recommended. However, given the almost complete consistency between the results of t-test and Mann-Whitney $U$ test, even if the sample size is less than 30 people, using t-test seems appropriate.

Wilcoxon test results based on the scores obtained by patients showed that participants' responses were significantly different before and after treatment protocol using rTMS ( $<<0.001)$ (Table 2).

According to the results of Chi-Square tests, none of the underlying variables including sex $(\mathrm{p}=0.540)$, hypertension $(p=0.999)$, diabetes $(p=0.999)$, dementia $(p=0.999)$, and hemiplegia $(p=0.773)$ had a significant relationship with response to treatment protocol using rTMS.

\section{Discussion}

The findings confirmed the hypothesis that using repetitive Transcranial Magnetic Stimulation (rTMS) through low-frequency stimulation (one $\mathrm{Hz}$ ) in Broca's area of the right hemisphere is effective on patients' motor aphasia treatment and improved dysarthria after CVA. The results of data analysis showed a significant difference between patients' test results before and after Cerebrovascular Accident (CVA).

Hamilton et al. (7), in their study on a person with chronic motor (non-fluent) aphasia that received 1200 pulses of rTMS daily at a frequency of one hertz for 10 days in Broca's area in the right hemisphere, concluded that cortical stimulation in healthy side (the right hemisphere) in contralesional side in patients with motor aphasia may improve speech. This result is consistent with the result of the present study as rTMS improved aphasia caused by CVA.

The findings of Medina et al. (16) on eight patients with CVA in MCA area in the left hemisphere with mild to moderate motor aphasia showed that rTMS significantly increased speech productivity compared to the baseline.

Table 1. Education status of participants based on their gender

\begin{tabular}{lcccccc}
\hline Education Status & \multicolumn{2}{c}{ Men } & \multicolumn{2}{c}{ Women } & \multicolumn{2}{c}{ Total } \\
\cline { 2 - 7 } & Frequency & Percent & Frequency & Percent & Frequency & Percent \\
Under Diploma & 9 & 52.9 & 6 & 85.7 & 15 & 62.5 \\
Diploma & 3 & 17.6 & 1 & 14.3 & 4 & 16.7 \\
Master's Degree & 5 & 29.4 & - & 0.0 & 5 & 20.8 \\
Total & 17 & 100.0 & 7 & 100.0 & 24 & 100.0 \\
\hline
\end{tabular}

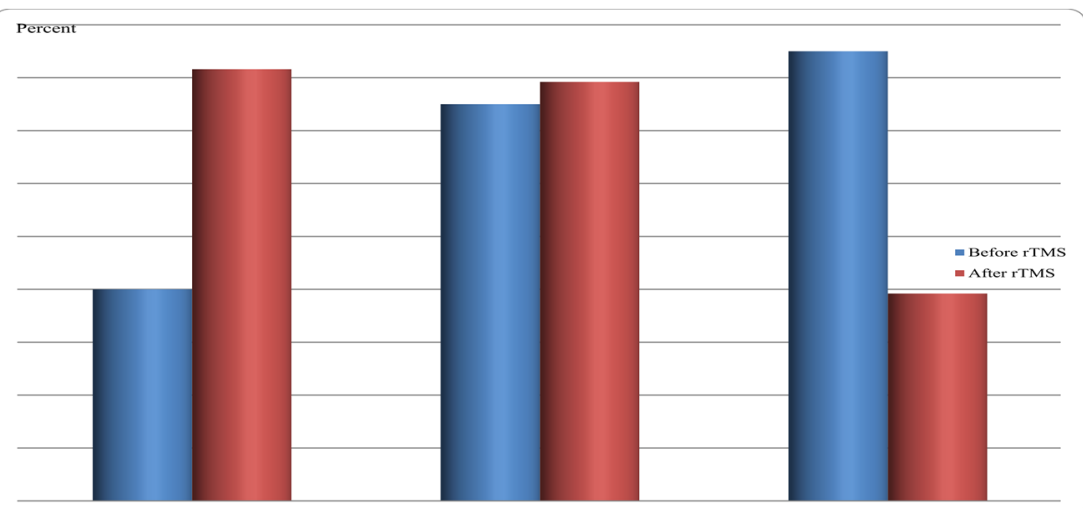

Diagram 1. The status of patients' response $(n=24)$ to the images before and after treatment protocol using rTMS

Table 2. Mean, SD and Wilcoxon test result of patients' responses to images before and after treatment protocol using rTMS

\begin{tabular}{lccc}
\hline rTMS & Mean & Std. Deviation & Wilcoxon Test Result \\
\hline Before & 7.1 & 75.51 & $\mathrm{Z}=-4.351$ \\
After & 12.1 & 12.19 & $\mathrm{P}<0.001$ \\
\hline
\end{tabular}


However, no significant increase was found in the sentence productivity or grammar accuracy. These results suggest that rTMS of Broca's area of the cerebral cortex (IFG) in the right hemisphere in patients with chronic motor aphasia may improve speech fluency, but it may not be effective on other aspects of language production. This study is also consistent with the above-mentioned study in terms of increased verbal productivity after using rTMS.

Barwood et al. (17) used 20 minutes of low-frequency rTMS (one $\mathrm{Hz}$ ) on the anterior portion of Broca's area (right hemisphere) for 10 days in 12 patients with motor aphasia, two to six years after CVA. Electrophysiological results indicated rTMS capacity in regulating neural language networks and lexical - semantic performance in participants with motor aphasia. They suggested that time may be an important factor in the reorganization of brain neurons following rTMS. These results can confirm the effect of rTMS on the verbal improvement of aphasia patients.

Barwood et al. (18) used 20 minutes of low-frequency rTMS (one $\mathrm{Hz}$ ) for six patients with actual stimulation and six patients with sham placebo for 10 days. The results showed that treatment-related changes were observed two months after stimulation in the stimulation group compared to placebo-sham group in naming objects and also other significant aspects of language and aural comprehension (in the sensory aphasia). The results of the mentioned study are consistent with the findings of the present study.

Naeser et al. (11) used ten minutes of rTMS at a frequency of one hertz per second for inhibitory stimulation of the anterior region of Broca's area in the right hemisphere in eight aphasia patients. In aphasia patients, inhibition of the anterior triangular region of Broca's area in the right hemisphere (pars triangularis (PTr)) led to a significant increase in naming images and a significant reduction in response time (RT). Inhibition of the right pars opercularis (POp) led to a significant increase in RT. However, no change was observed in the number of naming images. Eight normal people similar to aphasia patients mentioned the name of all images correctly, and RT significantly reduced following rTMS to inhibit the right PTr versus the right POp. Differential effects of inhibition of the right PTr versus right POp indicate different functional roles in these areas.

In a randomized controlled study by Weiduschat et al. (19), the effect of one Hertz rTMS on Broca's area in the right hemisphere in front of the lesion in aphasia patients after CVA was studied in an acute phase. According to their group allocation, the patients received several sessions of rTMS on the RT PTr in addition to conventional speech therapy (intervention group) or on the vertex (control group). The intervention group significantly improved clinically in the Aachen aphasia test total score $(p=0.002)$, while the control group did not.

In the study of Barwood and Murdoch (20), active stimulation using 20 minutes of low-frequency rTMS (one $\mathrm{Hz}$ ) was conducted for six patients and placebo for six other patients for 10 days. The study findings showed treatmentrelated changes in which improved performance of speech, aural comprehension and fluent expression were observed in the stimulation group compared to the control (placebo) group during 12 months of stimulation; the findings confirm the results of the present study in terms of the effect of rTMS on improved speech performance of aphasia patients.

Davidson (21), mentioned that integration of noninvasive cortical stimulation (NICS) (tDCS/rTMS) with speech therapy can improve aural comprehension abilities in aphasia patients after stroke in the left hemisphere. The results of several studies provide explanatory evidence for integration of speech therapy and NICS. Others suggest that combination of NICS and speech interventions will improve aural comprehension compared to speech therapy alone. The literature provides explanatory evidence about the effectiveness of NICS with speech therapy in improved aural comprehension ability in aphasia patients after stroke in the left hemisphere. Although the above review study shows the effectiveness of using TMS in improved aural comprehension, it is not exactly consistent with the results of the present study, as we did not use speech therapy simultaneously with rTMS.

\section{Conclusion}

The results of data analysis showed that low-frequency rTMS in Broca's area in the right hemisphere was effective on improved dysarthria in patients with motor aphasia caused by CVA. The results of many studies are consistent with our results. According to the studies, it can be concluded that low-frequency rTMS would be effective in verbal improvement and reducing the symptoms of aphasia after stroke, and it can be recommended for neurologists and decision-makers to choose the method to treat patients with aphasia caused by CVA.

It must be noted that the generalization of the results of this study is limited to right-handed people and it seems that similar studies with a larger sample size can increase the generalizability of the results. Also, it should be mentioned that sham control, used as a standard placebo of TMS, did not exist in Iran at the time of the study. Therefore, selecting a control group for this study was not possible, which is considered as a limitation of this study.

\section{Acknowledgement}

Hereby, we greatly appreciate the cooperation and assistance provided by the Research Deputy of Shahid Beheshti University of Medical Sciences for providing financial resources and facilities necessary to conduct this project, also all staff in Loghman Hakim Hospital and the specialty clinic.

Details of this research project are available in the Iranian Registry of Clinical Trials (IRCT). The registration ID in IRCT is IRCT2014052417814N1. The researchers did not have any conflict of interest in this study.

\section{Conflict of Interests}

The authors declare that they have no competing interests. 


\section{References}

1. Boram L, Sun-Bom P. Characteristics of cognitive impairment in patients with post-stroke aphasia. Ann Rehabil Med. 2014;38(6):75965 .

2. Musser B, Wilkinson J, Gilbert T, Bokhour BG. Changes in identity after aphasic stroke: Implications for primary care. Int J Family Med. 2015:1-8.

3. Tippett DC NJ, Hillis AE. Aphasia: Current concepts in theory and practice. J Neurol Transl Neurosci. 2014;2(1):1-7.

4. Soltani S, Khatoonabadi AR, Jenabi MS, Piran A. Frequency of aphasia resulting from stroke at hospitals affiliated to Tehran University of Medical Sciences. J Modern Rehabil. 2013;6(4):44-8. [In Persian]

5. Hamilton RF, Chrysikou EG, Coslett B. Mechanisms of aphasia recovery after stroke and the role of noninvasive brain stimulation. Brain Lang. 2011;118(1-2):40-50.

6. Shah-Basak P, Norise C, Garcia G, Torres J, Faseyitan O, Hamilton $\mathrm{R}$. Individualized treatment with transcranial direct current stimulation in patients with chronic non-fluent aphasia due to stroke. Front Hum Neurosci. 2015;9:201.

7. Hamilton RH, Sanders L, Benson J, Faseyitan O, Norise C, Naeser $\mathrm{M}$, et al. Stimulating conversation: Enhancement of elicited propositional speech in a patient with chronic non-fluent aphasia following transcranial magnetic stimulation. Brain Lang. 2010;113(1):45-50.

8. Naeser MA, Martin PI, Ho M, Treglia E, Kaplan E, Bashir S, et al. Transcranial magnetic stimulation and aphasia rehabilitation. Arch Phys Med Rehabil. 2012;93(1 Suppl):S26-34.

9. Naeser MA, Martin PI, Theoret H, Kobayashi M, Fregni F, Nicholas $\mathrm{M}$, et al. TMS suppression of right pars triangularis, but not pars opercularis, improves naming in aphasia. Brain Lang. 2011;119(3):206-13.

10. Barwood CHS, Murdoch BE, Whelan BM, Lloyd D, Riek S, O' Sullivan JD, et al. Improved language performance subsequent to low-frequency rTMS in patients with chronic non-fluent aphasia poststroke. Eur J Neurol. 2011;18(7):935-43.

11. Szaflarski JP, Vannest J, Wu SW, Difrancesco MW, Banks C, Gilbert DL. Excitatory repetitive transcranial magnetic stimulation induces improvements in chronic post-stroke aphasia. Med Sci Monit 2011;17(3):CR132-9.

12. Torres J, Drebing D, Hamilton R. TMS and tDCS in post-stroke aphasia: Integrating novel treatment approaches with mechanisms of plasticity. Restor Neurol Neurosci. 2013;31(4):501-15.

13. Seniow J WK, Lesniak M, Iwanski S, Czepiel W, Cztonkowska A. Transcranial Magnetic Stimulation combined with speech and language training in early aphasia rehabilitation: A randomized double-blind controlled pilot study. To Stroke Rehabil. 2013;20(3):250-61

14. Ren CL, Zhang GF, Xia N, Jin CH, Zhang XH, Hao JF, et al. Effect of low-frequency rTMS on aphasia in stroke patients: a meta-analysis of randomized controlled trials. PLoS One. 2014; 9(7):e102557.

15. Wong IS1, Tsang HW. A review on the effectiveness of repetitive transcranial magnetic stimulation (rTMS) on post-stroke aphasia. Rev Neurosci. 2013; 24(1):105-14.

16. Medina JHR, Norise C, Turkeltaub PE, Coslett HB. Transcranial Magnetic Stimulation improves discourse productivity in individuals with non-fluent aphasia. Procedia Soc Behav Sci. 2011;23:167-8.

17. Barwood CHS, Murdoch BE, Whelan BM, Lloyd D, Riek S, O'Sullivan JD, et al. Modulation of N400 in chronic non-fluent aphasia using low frequency Repetitive Transcranial Magnetic Stimulation (rTMS). Brain Lang. 2011;116(3):125-35.

18. Barwood CHS, Murdoch BE, Whelan BM, Lloyd D, Riek S, Sullivan JDO, et al. Improved language performance subsequent to low-frequency rTMS in patients with chronic non-fluent aphasia poststroke. Eur J Neurol. 2011;18(7):935-43.

19. Weiduschat N, Thiel A, Rubi-Fessen I, Hartmann A, Kessler J, Merl $\mathrm{P}$, et al. Effects of Repetitive Transcranial Magnetic Stimulation in aphasic stroke a randomized controlled pilot study. Stroke. 2011;42(2):409-15.

20. Barwood CHS, Murdoch BE. rTMS as a treatment for neurogenic communication and swallowing disorders. Acta Neurol Scand 2013;127(2):77-91.

21. Davidson A. Are therapeutic interventions that include non-invasive brain stimulation (tDCS/rTMS) with language therapy effective at improving auditory comprehension in patients with aphasia following left-hemisphere stroke? [Critical Review]. 2014, https://www.uwo.ca/fhs/ lwm/teaching/EBP/2013-14/Davidson.pdf. Western University: School of Communication Sciences and Disorders. 